\title{
From Twisted Quantum Loop Algebras to Twisted Yangians ${ }^{\star}$
}

\author{
Patrick CONNER ${ }^{\dagger}$ and Nicolas GUAY ${ }^{\ddagger}$ \\ $\dagger$ Science Department, Red Deer College, Red Deer, Alberta T4N 5H5, Canada \\ E-mail: patrick.conner@rdc.ab.ca \\ $\ddagger$ Department of Mathematical and Statistical Sciences, University of Alberta, \\ CAB 632, Edmonton, Alberta T6G 2G1, Canada \\ E-mail: nguay@ualberta.ca \\ URL: http://www.ualberta.ca/ nguay/
}

Received January 30, 2015, in final form May 01, 2015; Published online May 14, 2015

http://dx.doi.org/10.3842/SIGMA.2015.040

\begin{abstract}
We prove how the Yangian of $\mathfrak{g l}_{N}$ in its RTT presentation and Olshanski's twisted Yangians for the orthogonal and symplectic Lie algebras can be obtained by a degeneration process from the corresponding quantum loop algebra and some of its twisted analogues.
\end{abstract}

Key words: twisted Yangians; twisted quantum loop algebras; degeneration; RTT-presentation

2010 Mathematics Subject Classification: 17B37; 16T20; 81R50

\section{Introduction}

Yangians are quantum groups which appeared over thirty years ago. Since that time, it has been shown that they play a role in the theory of integrable systems by controlling some of their symmetries $[2,4,6,20,25,26]$. It has been discovered that, for integrable systems with boundary, symmetries are better captured by twisted Yangians, which are coideal subalgebras of Yangians [10, 11, 22, 23, 24]. This has provided motivation to mathematicians to study the representation theory of Yangians and twisted Yangians: see [27] and references therein, in particular the work of A. Molev and M. Nazarov.

It has been well known for a long time that quantum loop algebras and Yangians associated to complex semisimple Lie algebras have similar representation theories although, until very recently, explicit connections between their module categories (other than just analogies and similarities) were not available. The papers $[15,16]$ seem to be the first ones to establish rigorously important equivalences between certain categories of modules for both of those quantum groups.

It is also commonly known that Yangians are limit forms of quantum loop algebras: this was explicitly stated in [13], but a proof appeared only much later in [18] although such a proof was certainly known to some experts. (One early result in that direction can be found in Appendix C in [3].) Stronger results were obtained at about the same time by V. Toledano Laredo and S. Gautam: see [17]. The results of these two papers apply to Yangians associated to complex semisimple Lie algebras presented using what is commonly called Drinfeld's second realization [14]. Our first goal in this article is to prove that the Yangian of $\mathfrak{g l}_{N}$ is isomorphic to a limit form $\widetilde{Y}\left(\mathfrak{g l}_{N}\right)$ of the quantum loop algebra of $\mathfrak{g l}_{N}$ using the generators from the

\footnotetext{
${ }^{\star}$ This paper is a contribution to the Special Issue on Exact Solvability and Symmetry Avatars in honour of Luc Vinet. The full collection is available at http://www.emis.de/journals/SIGMA/ESSA2014.html
} 
RTT-presentation. (Some indications about how to show this are given in [9], but we follow a different approach.) It is not clear if this could be used to deduce the main theorem of [18] in the $\mathfrak{s l}_{n}$-case or vice-versa: this would probably require checking that the isomorphisms in the main theorem of [18] and in Theorem 2.2 below are compatible with the isomorphisms between the two realizations of quantum loop algebras and Yangians for $\mathfrak{g l}_{n}$ established in [12] and [5].

In the second half of [18], it was established that certain degenerate forms of twisted quantum loop algebras associated to a complex semisimple Lie algebra $\mathfrak{g}$ and a Dynkin diagram automorphism of $\mathfrak{g}$ are isomorphic to the ordinary Yangian of $\mathfrak{g}$. This explains partially why there is no known twisted Yangian attached to such data in general. The two cases considered in section 3 below are however different: the twisted quantum loop algebras of type AI and AII are associated to an involution $\theta$ of $\mathfrak{g l}_{N}$ and are quantizations of the enveloping algebra of the twisted loop algebra which is the fixed point of the involution $\Theta$ on $\mathfrak{g l}_{N} \otimes_{\mathbb{C}} \mathbb{C}\left[s, s^{-1}\right]$ given by $\Theta(X \otimes s)=\theta(X) \otimes s^{-1}$, not $\Theta(X \otimes s)=-\theta(X) \otimes s$ as would be the case with a twisted affine Kac-Moody algebra associated to a Dynkin diagram automorphism $\theta$ of order 2. Theorem 3.1 states that the isomorphism $\varphi$ of Theorem 2.2 restricts to the twisted Yangian $Y^{t w}\left(\mathfrak{g}_{N}\right)$ $\left(\mathfrak{g}_{N}=\mathfrak{o}_{N}\right.$ or $\left.\mathfrak{g}_{N}=\mathfrak{s p}_{N}\right)$, viewed as a subalgebra of $Y\left(\mathfrak{g l}_{N}\right)$, and provides an isomorphism with a certain subalgebra of $\widetilde{Y}\left(\mathfrak{g l}_{N}\right)$ obtained from the generators of the corresponding twisted quantum loop algebra. Theorem 3.2 says that $Y^{t w}\left(\mathfrak{g}_{N}\right)$ is isomorphic to a certain limit form of the twisted quantum loop algebra and is analogous to Theorem 2.2 for $Y\left(\mathfrak{g l}_{N}\right)$. Results similar to Theorems 3.1 and 3.2 should hold for the twisted quantum loop algebras and twisted Yangians of type AIII treated in [8] (resp. [28]), and for the twisted quantum loop superalgebra and twisted super-Yangians explored in [7] (resp. [30]). When $\mathfrak{g}$ is a classical simple Lie algebra other than $\mathfrak{s l}_{n}$, there is also a way to present its Yangian $Y(\mathfrak{g})$ using using an RTT-relation for some appropriate matrix $R$, see [1]. (In loc. cit., $Y(\mathfrak{g})$ is obtained as a quotient of what the authors call the extended Yangian $X(\mathfrak{g})$.) The main results of [1] should admit analogues for quantum loop algebras, but the present authors ignore whether this has been worked out or not. If so, then Theorem 2.2 below should have an analogue for Yangians and quantum loop algebras attached to $\mathfrak{g}$ via RTT-relations when $\mathfrak{g}$ is of classical Dynkin type. Furthermore, twisted Yangians which are coideal subalgebras of $Y(\mathfrak{g})$ and of $X(\mathfrak{g})$ have been studied recently in [19] and hopefully admit $q$-analogues for which a version of Theorem 3.2 should hold also. As for more general twisted Yangians associated to an arbitrary symmetric pair (see [24] for instance), it is reasonable to think that they are also limit forms of certain twisted quantum loop algebras: the quantum symmetric Kac-Moody pairs introduced in [21] may be relevant here.

One motivation for this paper is that it should help in establishing an isomorphism using the RTT-generators between completions of the Yangian and quantum loop algebra of $\mathfrak{g l}_{n}$ similar to the one obtained in [17] using the generators from Drinfeld's second realization. Once such a result is obtained, one may hope that such an isomorphism would work also for twisted Yangians and twisted quantum loop algebras. Eventually, the goal would be to establish equivalences between module categories for twisted Yangians and twisted quantum loop algebras as in $[15,16]$.

\section{Quantum loop algebra and Yangian for $\mathfrak{g l}_{N}$}

We start by recalling the definition of the Yangian and of the quantum loop algebra of $\mathfrak{g l}_{N}$ in the RTT presentation [32]. We view $\hbar$ as a formal parameter.

Definition 2.1. Let $R(u)=1 \otimes 1-\hbar u^{-1} \sum_{i, j=1}^{N} E_{i j} \otimes E_{j i}$ where $E_{i j}$ denotes the usual elementary matrix. The Yangian $Y\left(\mathfrak{g l}_{N}\right)$ is the unital associative algebra over $\mathbb{C}[\hbar]$ generated by elements $t_{i j}^{(r)}$ for $r \in \mathbb{Z}_{+}, i, j \in\{1, \ldots, N\}$ satisfying $t_{i j}^{(0)}=\delta_{i j}$, together with the following relations: if 


$$
\begin{gathered}
t_{i j}(u)=\sum_{r=0}^{\infty} t_{i j}^{(r)} u^{-r} \in Y\left(\mathfrak{g l}_{N}\right)\left[\left[u^{-1}\right]\right] \text { and } t(u)=\sum_{i, j=1}^{N} E_{i j} \otimes t_{i j}(u), \text { then } \\
R(u-v) t_{1}(u) t_{2}(v)=t_{2}(v) t_{1}(u) R(u-v)
\end{gathered}
$$

where $t_{1}(u)\left(\right.$ resp. $\left.t_{2}(u)\right)$ is the element of $\operatorname{End}_{\mathbb{C}}\left(\mathbb{C}^{N}\right) \otimes_{\mathbb{C}} \operatorname{End}_{\mathbb{C}}\left(\mathbb{C}^{N}\right) \otimes_{\mathbb{C}} Y\left(\mathfrak{g l}_{N}\right)\left[\left[u^{-1}\right]\right]$ obtained by replacing $E_{i j}$ by $E_{i j} \otimes I$ (resp. $\left.I \otimes E_{i j}\right)$ in $t(u)$. Here, we have also identified $R(u-v)$ with $R(u-v) \otimes 1$. Relation (2.1) is equivalent to the system of relations

$$
\left[t_{i j}^{(m+1)}, t_{k l}^{(n)}\right]-\left[t_{i j}^{(m)}, t_{k l}^{(n+1)}\right]=\hbar\left(t_{k j}^{(m)} t_{i l}^{(n)}-t_{k j}^{(n)} t_{i l}^{(m)}\right) .
$$

Definition 2.2. The quantum affine $R$-matrix is the element of $\operatorname{End}_{\mathbb{C}}\left(\mathbb{C}^{N}\right) \otimes \operatorname{End}_{\mathbb{C}}\left(\mathbb{C}^{N}\right) \otimes_{\mathbb{C}}$ $\mathbb{C}[u, v]$ given by

$$
\begin{aligned}
R_{q}(u, v)= & \sum_{i, j=1}^{N}\left(u q^{-\delta_{i j}}-v q^{\delta_{i j}}\right) E_{i i} \otimes E_{j j}-\left(q-q^{-1}\right) u \sum_{\substack{i, j=1 \\
i>j}}^{N} E_{i j} \otimes E_{j i} \\
& -\left(q-q^{-1}\right) v \sum_{\substack{i, j=1 \\
i<j}}^{N} E_{i j} \otimes E_{j i} .
\end{aligned}
$$

Set $\mathcal{L}\left(\mathfrak{g l}_{N}\right)=\mathfrak{g l}_{N} \otimes_{\mathbb{C}} \mathbb{C}\left[s, s^{-1}\right]$.

Definition 2.3. The quantum loop algebra $\mathfrak{U}_{q}\left(\mathcal{L}\left(\mathfrak{g l}_{N}\right)\right)$ is the unital associative algebra over $\mathbb{C}(q)$ generated by $\left\{T_{i j}^{(r)}, \bar{T}_{i j}^{(r)} \mid r \in \mathbb{Z}_{+}, i, j=1, \ldots, N\right\}$ which must satisfy the following relations

$$
\begin{aligned}
& T_{i j}^{(0)}=0=\bar{T}_{j i}^{(0)} \quad \text { if } \quad 1 \leq i<j \leq N, \\
& T_{i i}^{(0)} \bar{T}_{i i}^{(0)}=1=\bar{T}_{i i}^{(0)} T_{i i}^{(0)} \quad \forall 1 \leq i \leq N, \\
& R_{q}(u, v) T_{1}(u) T_{2}(v)=T_{2}(v) T_{1}(u) R_{q}(u, v), \\
& R_{q}(u, v) \bar{T}_{1}(u) \bar{T}_{2}(v)=\bar{T}_{2}(v) \bar{T}_{1}(u) R_{q}(u, v), \\
& R_{q}(u, v) \bar{T}_{1}(u) T_{2}(v)=T_{2}(v) \bar{T}_{1}(u) R_{q}(u, v),
\end{aligned}
$$

$T_{a}(u)$ and $\bar{T}_{a}(u)$ are obtained from $T(u):=\sum_{i, j=1}^{N} E_{i j} \otimes T_{i j}(u)$ and $\bar{T}(u):=\sum_{i, j=1}^{N} E_{i j} \otimes \bar{T}_{i j}(u)$ in the same way as $t_{a}(u)$ in Definition 2.1; in this case, $T_{i j}(u), \bar{T}_{i j}(u)$ are the elements

$$
T_{i j}(u)=\sum_{r=0}^{\infty} T_{i j}^{(r)} u^{-r} \in \mathfrak{U}_{q}\left(\mathcal{L}\left(\mathfrak{g l}_{N}\right)\right)\left[\left[u^{-1}\right]\right], \quad \bar{T}_{i j}(u)=\sum_{r=0}^{\infty} \bar{T}_{i j}^{(r)} u^{r} \in \mathfrak{U}_{q}\left(\mathcal{L}\left(\mathfrak{g l}_{N}\right)\right)[[u]] .
$$

Relations (2.2),(2.3) and (2.4) can be made more explicit in terms of the generators of $\mathfrak{U}_{q}\left(\mathcal{L}\left(\mathfrak{g l}_{N}\right)\right)$. For instance, (2.2) is equivalent to the following family of relations

$$
\begin{aligned}
\left(q^{-\delta_{i k}}\right. & \left.T_{i j}^{(r+1)} T_{k l}^{(s)}-q^{\delta_{i k}} T_{i j}^{(r)} T_{k l}^{(s+1)}\right)-\left(q^{-\delta_{j l} l} T_{k l}^{(s)} T_{i j}^{(r+1)}-q^{\delta_{j l}} T_{k l}^{(s+1)} T_{i j}^{(r)}\right) \\
= & \left(q-q^{-1}\right)\left(\delta_{i>k} T_{k j}^{(r+1)} T_{i l}^{(s)}+\delta_{i<k} T_{k j}^{(r)} T_{i l}^{(s+1)}\right) \\
& -\left(q-q^{-1}\right)\left(\delta_{l>j} T_{k j}^{(s)} T_{i l}^{(r+1)}+\delta_{l<j} T_{k j}^{(s+1)} T_{i l}^{(r)}\right) .
\end{aligned}
$$

Let $\mathcal{A}$ be the localization of $\mathbb{C}\left[q, q^{-1}\right]$ at the ideal $(q-1)$. Let $\mathfrak{U}_{\mathcal{A}}\left(\mathcal{L}\left(\mathfrak{g l}_{N}\right)\right)$ be the $\mathcal{A}$-subalgebra of $\mathfrak{U}_{q}\left(\mathcal{L}\left(\mathfrak{g l}_{N}\right)\right)$ generated by the elements $\tau_{i j}^{(r)}, \bar{\tau}_{i j}^{(r)}$ given by

$$
\tau_{i j}^{(r)}=\frac{T_{i j}^{(r)}}{q-q^{-1}}, \quad \bar{\tau}_{i j}^{(r)}=\frac{\bar{T}_{i j}^{(r)}}{q-q^{-1}} \quad \text { for } \quad r \geq 0, \quad 1 \leq i, j \leq N,
$$


except that, when $r=0$ and $i=j$, we set

$$
\tau_{i i}^{(0)}=\frac{T_{i i}^{(0)}-1}{q-1}, \quad \bar{\tau}_{i i}^{(0)}=\frac{\bar{T}_{i i}^{(0)}-1}{q-1} .
$$

Theorem 2.1 (Section 3 of [29]). The assignment $E_{i j} s^{r} \mapsto \tau_{i j}^{(r)} \forall r \geq 0,1 \leq i, j \leq n$ except if $r=0$ and $1 \leq i<j \leq n, E_{i j} s^{-r} \mapsto-\bar{\tau}_{i j}^{(r)} \forall r \geq 0,1 \leq i, j \leq n$ except if $r=0$ and $1 \leq j<i \leq n$, induces an isomorphism $\mathfrak{U}\left(\mathcal{L}\left(\mathfrak{g l}_{N}\right)\right) \stackrel{\sim}{\longrightarrow} \mathfrak{U}_{\mathcal{A}}\left(\mathcal{L}\left(\mathfrak{g l}_{N}\right)\right) \otimes_{\mathcal{A}} \mathbb{C}$, where $\mathbb{C}$ is viewed as an $\mathcal{A}$-module via $\mathcal{A} /(q-1) \stackrel{\sim}{\longrightarrow} \mathbb{C}$.

We have the following sequence of algebra homomorphisms similar to the one considered in $[18]$

$$
\mathfrak{U}_{\mathcal{A}}\left(\mathcal{L}\left(\mathfrak{g l}_{N}\right)\right) \rightarrow \mathfrak{U}_{\mathcal{A}}\left(\mathcal{L}\left(\mathfrak{g l}_{N}\right)\right) /(q-1) \mathfrak{U}_{\mathcal{A}}\left(\mathcal{L}\left(\mathfrak{g l}_{N}\right)\right) \stackrel{\sim}{\longrightarrow} \mathfrak{U}\left(\mathcal{L}\left(\mathfrak{g l}_{N}\right)\right) \stackrel{s \mapsto 1}{\rightarrow} \mathfrak{U}\left(\mathfrak{g l}_{N}\right) .
$$

For $m \geq 0$, denote by $\mathrm{K}_{m}$ the Lie ideal of $\mathcal{L}\left(\mathfrak{g l}_{N}\right)$ spanned by $X \otimes s^{r}(s-1)^{m} \forall r \in \mathbb{Z}, \forall X \in \mathfrak{g l}_{N}$. Let $U$ be the subspace of $\mathfrak{U}_{\mathcal{A}}\left(\mathcal{L}\left(\mathfrak{g l}_{N}\right)\right)$ spanned over $\mathbb{C}$ by the generators $\tau_{i j}^{(r)}, \bar{\tau}_{i j}^{(r)}$ and observe that

$$
U \cap(q-1) \mathfrak{U}_{\mathcal{A}}\left(\mathcal{L}\left(\mathfrak{g l}_{N}\right)\right)=\operatorname{span}_{\mathbb{C}}\left\{\tau_{i i}^{(0)}+\bar{\tau}_{i i}^{(0)} \mid i=1, \ldots, N\right\} .
$$

This is because $(q-1) \mathfrak{U}_{\mathcal{A}}\left(\mathcal{L}\left(\mathfrak{g l}_{N}\right)\right)=\operatorname{Ker}(\psi)$, where $\psi$ is the composite

$$
\mathfrak{U}_{\mathcal{A}}\left(\mathcal{L}\left(\mathfrak{g l}_{N}\right)\right) \rightarrow \mathfrak{U}_{\mathcal{A}}\left(\mathcal{L}\left(\mathfrak{g l}_{N}\right)\right) /(q-1) \mathfrak{U}_{\mathcal{A}}\left(\mathcal{L}\left(\mathfrak{g l}_{N}\right)\right) \stackrel{\sim}{\longrightarrow} \mathfrak{U}\left(\mathcal{L}\left(\mathfrak{g l}_{N}\right)\right) .
$$

Moreover, notice that

$$
\tau_{i i}^{(0)}+\bar{\tau}_{i i}^{(0)}=\frac{\bar{T}_{i i}^{(0)}\left(T_{i i}^{(0)}-1\right)^{2}}{q-1}=(q-1)\left((q-1) \bar{\tau}_{i i}^{(0)}+1\right)\left(\tau_{i i}^{(0)}\right)^{2} \in(q-1) \mathfrak{U}_{\mathcal{A}}\left(\mathcal{L}\left(\mathfrak{g l}_{N}\right)\right) .
$$

Let $\mathbf{K}_{0}=\mathfrak{U}_{\mathcal{A}}\left(\mathcal{L}\left(\mathfrak{g l}_{N}\right)\right)$. For $m \geq 1$, let $\mathbb{K}_{m}$ be the two-sided ideal of $\mathfrak{U}_{\mathcal{A}}\left(\mathcal{L}\left(\mathfrak{g l}_{N}\right)\right)$ generated by $\psi^{-1}\left(\mathrm{~K}_{m}\right) \cap U$, and set $\mathbf{K}_{m}$ to be the sum of the ideals $\left(q-q^{-1}\right)^{m_{0}} \mathbb{K}_{m_{1}} \cdots \mathbb{K}_{m_{k}}$ with $m_{0}+m_{1}+$ $\cdots+m_{k} \geq m$. This is slightly different from the definition of the analogous ideals $\mathbf{K}_{m}$ in [18] in the case of $\mathfrak{s l}_{N}$ because, for the $\mathfrak{g l}_{N}$ case, $\mathbf{K}_{1}^{m}$ is strictly smaller than $\mathbf{K}_{m}$.

Let $\widetilde{Y}\left(\mathfrak{g l}_{N}\right)$ be the $\mathbb{C}$-algebra

$$
\tilde{Y}\left(\mathfrak{g l}_{N}\right)=\bigoplus_{m=0}^{\infty} \mathbf{K}_{m} / \mathbf{K}_{m+1} .
$$

$\widetilde{Y}\left(\mathfrak{g l}_{N}\right)$ can be viewed as a $\mathbb{C}[\hbar]$-algebra if we set $\hbar=\overline{q-q^{-1}} \in \mathbf{K}_{1} / \mathbf{K}_{2}$. Note that the first quotient is isomorphic to $\mathfrak{U}\left(\mathfrak{g l}_{N}\right)$ by definition.

Theorem 2.2. $\widetilde{Y}\left(\mathfrak{g l}_{N}\right)$ is isomorphic to $Y\left(\mathfrak{g l}_{N}\right)$.

For the analogue of our first theorem for an arbitrary complex semisimple Lie algebra, see [13] and [18].

For $m, r \geq 0$, define recursively elements $T_{i j}^{(r, m)}$ in the following way

$$
T_{i j}^{(r, 0)}=\tau_{i j}^{(r)} \quad \text { and } \quad T_{i j}^{(r, m+1)}=T_{i j}^{(r+1, m)}-T_{i j}^{(r, m)},
$$

except that if $i<j, T_{i j}^{(0,0)}=-\bar{\tau}_{i j}^{(0)}$.

One can check by induction on $m$ that, for every $r, \psi\left(T_{i j}^{(r, m)}\right)=E_{i j} s^{r}(s-1)^{m}$, hence $T_{i j}^{(r, m)} \in \mathbf{K}_{m}$. Set $\xi_{i j}^{(r, m)}=\overline{T_{i j}^{(r, m)}} \in \mathbf{K}_{m} / \mathbf{K}_{m+1}$. 
Proof of Theorem 2.2. We will prove that there is an isomorphism $\varphi: Y\left(\mathfrak{g l}_{N}\right) \stackrel{\sim}{\longrightarrow} \widetilde{Y}\left(\mathfrak{g l}_{N}\right)$ given by $t_{i j}^{(m+1)} \mapsto \xi_{i j}^{(0, m)}$ for $m \geq 0$.

Relation (2.5) can be rewritten in the following way

$$
\begin{aligned}
q^{-\delta_{i k}} & \left.T_{i j}^{(r+1)}-T_{i j}^{(r)}\right) T_{k l}^{(s)}-T_{i j}^{(r)}\left(q^{\delta_{i k}} T_{k l}^{(s+1)}-q^{-\delta_{i k}} T_{k l}^{(s)}\right) \\
& -\left(q^{-\delta_{j l}} T_{k l}^{(s)}\left(T_{i j}^{(r+1)}-T_{i j}^{(r)}\right)-\left(q^{\delta_{j l}} T_{k l}^{(s+1)}-q^{-\delta_{j l} l} T_{k l}^{(s)}\right) T_{i j}^{(r)}\right) \\
= & \left(q-q^{-1}\right)\left(\delta_{i>k} T_{k j}^{(r+1)} T_{i l}^{(s)}+\delta_{i<k} T_{k j}^{(r)} T_{i l}^{(s+1)}\right) \\
& -\left(q-q^{-1}\right)\left(\delta_{l>j} T_{k j}^{(s)} T_{i l}^{(r+1)}+\delta_{l<j} T_{k j}^{(s+1)} T_{i l}^{(r)}\right) .
\end{aligned}
$$

If $r, s \geq 1$ then, after rearranging and dividing both sides by $\left(q-q^{-1}\right)^{2}$, we get

$$
\begin{aligned}
q^{-\delta_{i k}} & \left.T_{i j}^{(r, 1)} T_{k l}^{(s, 0)}-T_{i j}^{(r, 0)} T_{k l}^{(s, 1)}\right)-\left(q^{\delta_{i k}}-q^{-\delta_{i k}}\right) T_{i j}^{(r, 0)} T_{k l}^{(s+1,0)} \\
& -q^{-\delta_{j l}}\left(T_{k l}^{(s, 0)} T_{i j}^{(r, 1)}-T_{k l}^{(s, 1)} T_{i j}^{(r, 0)}\right)+\left(q^{\delta_{j l}}-q^{-\delta_{j l}}\right) T_{k l}^{(s+1,0)} T_{i j}^{(r, 0)} \\
= & \left(q-q^{-1}\right)\left(\delta_{i>k} T_{k j}^{(r+1,0)} T_{i l}^{(s, 0)}+\delta_{i<k} T_{k j}^{(r, 0)} T_{i l}^{(s+1,0)}\right) \\
& -\left(q-q^{-1}\right)\left(\delta_{l>j} T_{k j}^{(s, 0)} T_{i l}^{(r+1,0)}+\delta_{l<j} T_{k j}^{(s+1,0)} T_{i l}^{(r, 0)}\right) .
\end{aligned}
$$

Using $T_{i j}^{(r+1, m)}-T_{i j}^{(r, m)}=T_{i j}^{(r, m+1)}$ and $T_{k l}^{(s+1, n)}-T_{k l}^{(s, n)}=T_{k l}^{(s, n+1)}$, we deduce by induction on $m$ and $n$ that, for all $r, s \geq 1$ and all $m, n \geq 0$,

$$
\begin{aligned}
q^{-\delta_{i k}} & \left.T_{i j}^{(r, m+1)} T_{k l}^{(s, n)}-T_{i j}^{(r, m)} T_{k l}^{(s, n+1)}\right)-\left(q^{\delta_{i k}}-q^{-\delta_{i k}}\right) T_{i j}^{(r, m)} T_{k l}^{(s+1, n)} \\
& -q^{-\delta_{j l}}\left(T_{k l}^{(s, n)} T_{i j}^{(r, m+1)}-T_{k l}^{(s, n+1)} T_{i j}^{(r, m)}\right)+\left(q^{\delta_{j l}}-q^{-\delta_{j l}}\right) T_{k l}^{(s+1, n)} T_{i j}^{(r, m)} \\
= & \left(q-q^{-1}\right)\left(\delta_{i>k} T_{k j}^{(r+1, m)} T_{i l}^{(s, n)}+\delta_{i<k} T_{k j}^{(r, m)} T_{i l}^{(s+1, n)}\right) \\
& -\left(q-q^{-1}\right)\left(\delta_{l>j} T_{k j}^{(s, n)} T_{i l}^{(r+1, m)}+\delta_{l<j} T_{k j}^{(s+1, n)} T_{i l}^{(r, m)}\right) .
\end{aligned}
$$

Consider the case $r=s=1$ in (2.6). Using $T_{i j}^{(r+1, m)}=T_{i j}^{(r, m+1)}+T_{i j}^{(r, m)}$ and $T_{k l}^{(r+1, n)}=$ $T_{k l}^{(r, n+1)}+T_{k l}^{(r, n)}$ for all $r \geq 0$, we obtain, for all $m, n \geq 0$,

$$
\begin{aligned}
q^{-\delta_{i k}}( & \left.T_{i j}^{(0, m+2)}+T_{i j}^{(0, m+1)}\right) T_{k l}^{(0, n+1)}-q^{-\delta_{i k}}\left(T_{i j}^{(0, m+1)}+T_{i j}^{(0, m)}\right) T_{k l}^{(0, n+2)} \\
& -\left(q^{\delta_{i k}}-q^{-\delta_{i k}}\right)\left(T_{i j}^{(0, m+1)}+T_{i j}^{(0, m)}\right) T_{k l}^{(1, n+1)}+q^{-\delta_{i k}}\left(T_{i j}^{(0, m+2)}+T_{i j}^{(0, m+1)}\right) T_{k l}^{(0, n)} \\
& -q^{-\delta_{i k}}\left(T_{i j}^{(0, m+1)}+T_{i j}^{(0, m)}\right) T_{k l}^{(0, n+1)}-\left(q^{\delta_{i k}}-q^{-\delta_{i k}}\right)\left(T_{i j}^{(0, m+1)}+T_{i j}^{(0, m)}\right) T_{k l}^{(1, n)} \\
& -q^{-\delta_{j l} l} T_{k l}^{(0, n+1)}\left(T_{i j}^{(0, m+2)}+T_{i j}^{(0, m+1)}\right)+q^{-\delta_{j l} l} T_{k l}^{(0, n+2)}\left(T_{i j}^{(0, m+1)}+T_{i j}^{(0, m)}\right) \\
& +\left(q^{\delta_{j l}}-q^{-\delta_{j l}}\right) T_{k l}^{(1, n+1)}\left(T_{i j}^{(0, m+1)}+T_{i j}^{(0, m)}\right)-q^{-\delta_{j l}} T_{k l}^{(0, n)}\left(T_{i j}^{(0, m+2)}+T_{i j}^{(0, m+1)}\right) \\
& +q^{-\delta_{j l}} T_{k l}^{(0, n+1)}\left(T_{i j}^{(0, m+1)}+T_{i j}^{(0, m)}\right)+\left(q^{\delta_{j l}}-q^{-\delta_{j l}}\right) T_{k l}^{(1, n)}\left(T_{i j}^{(0, m+1)}+T_{i j}^{(0, m)}\right) \\
= & \left(q-q^{-1}\right)\left(\delta_{i>k}\left(T_{k j}^{(1, m+1)}+T_{k j}^{(1, m)}\right) T_{i l}^{(0, n+1)}+\delta_{i<k}\left(T_{k j}^{(0, m+1)}+T_{k j}^{(0, m)}\right) T_{i l}^{(1, n+1)}\right) \\
& +\left(q-q^{-1}\right)\left(\delta_{i>k}\left(T_{k j}^{(1, m+1)}+T_{k j}^{(1, m)}\right) T_{i l}^{(0, n)}+\delta_{i<k}\left(T_{k j}^{(0, m+1)}+T_{k j}^{(0, m)}\right) T_{i l}^{(1, n)}\right) \\
& -\left(q-q^{-1}\right)\left(\delta_{l>j} T_{k j}^{(0, n+1)}\left(T_{i l}^{(1, m+1)}+T_{i l}^{(1, m)}\right)+\delta_{l<j} T_{k j}^{(1, n+1)}\left(T_{i l}^{(0, m+1)}+T_{i l}^{(0, m)}\right)\right) \\
& -\left(q-q^{-1}\right)\left(\delta_{l>j} T_{k j}^{(0, n)}\left(T_{i l}^{(1, m+1)}+T_{i l}^{(1, m)}\right)+\delta_{l<j} T_{k j}^{(1, n)}\left(T_{i l}^{(0, m+1)}+T_{i l}^{(0, m)}\right)\right) .
\end{aligned}
$$

Notice that both sides of this last equality are in $\mathbf{K}_{m+n+1}$ (and some of the terms are even in $\mathbf{K}_{m+n+2}$ or in $\mathbf{K}_{m+n+3}$ ). Modulo $\mathbf{K}_{m+n+2}$, we obtain the congruence

$$
q^{-\delta_{i k}}\left(T_{i j}^{(0, m+1)} T_{k l}^{(0, n)}-T_{i j}^{(0, m)} T_{k l}^{(0, n+1)}\right)-\left(q^{\delta_{i k}}-q^{-\delta_{i k}}\right) T_{i j}^{(0, m)} T_{k l}^{(1, n)}
$$




$$
\begin{aligned}
& -q^{-\delta_{j l}}\left(T_{k l}^{(0, n)} T_{i j}^{(0, m+1)}-T_{k l}^{(0, n+1)} T_{i j}^{(0, m)}\right)+\left(q^{\delta_{j l}}-q^{-\delta_{j l}}\right) T_{k l}^{(1, n)} T_{i j}^{(0, m)} \\
\equiv & \left(q-q^{-1}\right)\left(\delta_{i>k} T_{k j}^{(1, m)} T_{i l}^{(0, n)}+\delta_{i<k} T_{k j}^{(0, m)} T_{i l}^{(1, n)}\right) \\
& -\left(q-q^{-1}\right)\left(\delta_{l>j} T_{k j}^{(0, n)} T_{i l}^{(1, m)}+\delta_{l<j} T_{k j}^{(1, n)} T_{i l}^{(0, m)}\right) .
\end{aligned}
$$

Moreover, modulo $\mathbf{K}_{m+n+2}$, we also have

$$
\begin{aligned}
& \left(q^{\delta_{i k}}-q^{-\delta_{i k}}\right) T_{i j}^{(0, m)} T_{k l}^{(1, n)} \equiv\left(q^{\delta_{i k}}-q^{-\delta_{i k}}\right) T_{i j}^{(0, m)} T_{k l}^{(0, n)}, \\
& \left(q^{\delta_{j l}}-q^{-\delta_{j l}}\right) T_{k l}^{(1, n)} T_{i j}^{(0, m)} \equiv\left(q^{\delta_{j l}}-q^{-\delta_{j l}}\right) T_{k l}^{(0, n)} T_{i j}^{(0, m)}, \\
& \left(q-q^{-1}\right) T_{k j}^{(1, m)} T_{i l}^{(0, n)} \equiv\left(q-q^{-1}\right) T_{k j}^{(0, m)} T_{i l}^{(0, n)}, \\
& \left(q-q^{-1}\right) T_{k j}^{(0, m)} T_{i l}^{(1, n)} \equiv\left(q-q^{-1}\right) T_{k j}^{(0, m)} T_{i l}^{(0, n)}, \\
& \left(q-q^{-1}\right) T_{k j}^{(0, n)} T_{i l}^{(1, m)} \equiv\left(q-q^{-1}\right) T_{k j}^{(0, n)} T_{i l}^{(0, m)}, \\
& \left(q-q^{-1}\right) T_{k j}^{(1, n)} T_{i l}^{(0, m)} \equiv\left(q-q^{-1}\right) T_{k j}^{(0, n)} T_{i l}^{(0, m)} .
\end{aligned}
$$

Therefore, passing to the quotient $\mathbf{K}_{m+n+1} / \mathbf{K}_{m+n+2}$, we obtain

$$
\begin{aligned}
\left(\xi_{i j}^{(0, m+1)}\right. & \left.\xi_{k l}^{(0, n)}-\xi_{i j}^{(0, m)} \xi_{k l}^{(0, n+1)}\right)-\delta_{i k} \hbar \xi_{i j}^{(0, m)} \xi_{k l}^{(0, n)} \\
& \quad-\left(\xi_{k l}^{(0, n)} \xi_{i j}^{(0, m+1)}-\xi_{k l}^{(0, n+1)} \xi_{i j}^{(0, m)}\right)+\delta_{j l} \hbar \xi_{k l}^{(0, n)} \xi_{i j}^{(0, m)} \\
& =\hbar\left(\delta_{i>k} \xi_{k j}^{(0, m)} \xi_{i l}^{(0, n)}+\delta_{i<k} \xi_{k j}^{(0, m)} \xi_{i l}^{(0, n)}\right)-\hbar\left(\delta_{l>j} \xi_{k j}^{(0, n)} \xi_{i l}^{(0, m)}+\delta_{l<j} \xi_{k j}^{(0, n)} \xi_{i l}^{(0, m)}\right) .
\end{aligned}
$$

This last relation is equivalent to

$$
\left[\xi_{i j}^{(0, m+1)}, \xi_{k l}^{(0, n)}\right]-\left[\xi_{i j}^{(0, m)}, \xi_{k l}^{(0, n+1)}\right]=\hbar\left(\xi_{k j}^{(0, m)} \xi_{i l}^{(0, n)}-\xi_{k j}^{(0, n)} \xi_{i l}^{(0, m)}\right) .
$$

This holds for all $m, n \geq 0$.

All the previous computations prove that $\varphi: Y\left(\mathfrak{g l}_{N}\right) \longrightarrow \widetilde{Y}\left(\mathfrak{g l}_{N}\right)$ given by $\varphi\left(t_{i j}^{(m+1)}\right)=\xi_{i j}^{(0, m)}$ for $m \geq 0$ is an algebra homomorphism. We still have to show that $\varphi$ is injective and surjective.

We will first demonstrate surjectivity. Towards this end, we define elements $\bar{T}_{i j}^{(r, m)}$ as follows. Let $\bar{T}_{i j}^{(r, 0)}=\bar{\tau}_{i j}^{(r)}$, except that $\bar{T}_{i j}^{(0,0)}=-\tau_{i j}^{(0)}$ when $i \geq j$. Then, for each $m \geq 0$, let

$$
\bar{T}_{i j}^{(r, m+1)}=\bar{T}_{i j}^{(r+1, m)}-\bar{T}_{i j}^{(r, m)} .
$$

Also for each $m \geq 0$, let $\widetilde{T}_{i j}^{(0, m)}=\bar{T}_{i j}^{(0, m)}$ and $\widetilde{T}_{i j}^{(m, m)}=(-1)^{m+1} T_{i j}^{(0, m)}$, and for $1 \leq r \leq m$ define recursively

$$
\widetilde{T}_{i j}^{(r, m+1)}=\widetilde{T}_{i j}^{(r-1, m)}-\widetilde{T}_{i j}^{(r, m)} .
$$

Induction on $m$ shows that the elements $T_{i j}^{(r, m)}, \bar{T}_{i j}^{(r, m)}$ and $\widetilde{T}_{i j}^{(r, m)}$ respectively map via $\psi$ to the elements $E_{i j} s^{r}(s-1)^{m},(-1)^{m+1} E_{i j} s^{-(m+r)}(s-1)^{m}$ and $(-1)^{m+1} E_{i j} s^{-(m-r)}(s-1)^{m}$ in $\mathfrak{U}\left(\mathcal{L}\left(\mathfrak{g l}_{N}\right)\right)$. It follows that, for fixed $m$, the images of those elements under $\psi$ span $\mathrm{K}_{m}$. Moreover, all those elements are in $U$ by definition.

Note that for any fixed $X \in \psi^{-1}\left(\mathrm{~K}_{m}\right) \cap U$, there exists some element $Y$ in

$$
\operatorname{span}_{\mathbb{C}}\left\{T_{i j}^{(r, m)}, \bar{T}_{i j}^{(r, m)}, \widetilde{T}_{i j}^{(r, m)} \mid i, j=1, \ldots, N, r \in \mathbb{Z}_{+}\right\}
$$

for which $X-Y \in(q-1) \mathfrak{U}_{\mathcal{A}}\left(\mathcal{L}\left(\mathfrak{g l}_{N}\right)\right)$, because the map $\mathfrak{U}_{\mathcal{A}} \mathcal{L}\left(\mathfrak{g l}_{N}\right) /(q-1) \mathfrak{U}_{\mathcal{A}}\left(\mathcal{L}\left(\mathfrak{g l}_{N}\right)\right) \stackrel{\sim}{\longrightarrow}$ $\mathfrak{U}\left(\mathcal{L}\left(\mathfrak{g l}_{N}\right)\right)$ is an isomorphism. Since $X-Y$ is also in $U$, we have

$$
X-Y \in \operatorname{span}_{\mathbb{C}}\left\{\tau_{i i}^{(0)}+\bar{\tau}_{i i}^{(0)} \mid i=1, \ldots, N\right\} \subset \mathbf{K}_{\ell} \forall \ell \geq 0 .
$$

That $\tau_{i i}^{(0)}+\bar{\tau}_{i i}^{(0)}$ is in $\mathbf{K}_{\ell}$ for all $\ell \geq 0$ is a consequence of the fact that $\psi\left(\tau_{i i}^{(0)}+\bar{\tau}_{i i}^{(0)}\right)=0$. 
It follows that any element of $\mathbf{K}_{m}$ is congruent modulo $\mathbf{K}_{m+1}$ to a sum of monomials of the form $f(q)\left(q-q^{-1}\right)^{m_{0}} \mathcal{M}$ where $f(q) \in \mathcal{A}$ is not divisible by $q-1$ and $\mathcal{M}=\tau_{i_{1} j_{1}}^{\left(r_{1}, m_{1}\right)} \cdots \tau_{i_{k} j_{k}}^{\left(r_{k}, m_{k}\right)}$ with

$$
\tau_{i_{d} j_{d}}^{\left(r_{d}, m_{d}\right)} \in\left\{T_{i_{d} j_{d}}^{\left(r_{d}, m_{d}\right)}, \bar{T}_{i_{d} j_{d}}^{\left(r_{d}, m_{d}\right)}, \widetilde{T}_{i_{d} j_{d}}^{\left(r_{d}, m_{d}\right)}\right\}
$$

and $m_{0}+\cdots+m_{k} \geq m$. Moreover, since $T_{i_{d} j_{d}}^{\left(r_{d}, m_{d}\right)}-T_{i_{d} j_{d}}^{\left(r_{d}-1, m_{d}\right)}=T_{i_{d} j_{d}}^{\left(r_{d}-1, m_{d}+1\right)} \in \mathbb{K}_{m_{d}+1}$ (and similarly for $\bar{T}_{i_{d} j_{d}}^{\left(r_{d}, m_{d}\right)}$ and $\widetilde{T}_{i_{d} j_{d}}^{\left(r_{d}, m_{d}\right)}$ ), we can reduce modulo $\mathbf{K}_{m+1}$ to the case when $r_{d}=0$ for each $d=1, \ldots, k$.

Observe that modulo $\mathbb{K}_{m_{d}+1}$, we have

$$
\bar{T}_{i j}^{\left(0, m_{d}\right)} \equiv(-1)^{m_{d}+1} T_{i j}^{\left(0, m_{d}\right)} \equiv \widetilde{T}_{i j}^{\left(0, m_{d}\right)} .
$$

To see this, just take the difference of the elements on each side (this difference is in $U$ by definition) and apply $\psi$. Finally, observe that we can replace $f(q)$ by $f(1)$ modulo $\mathbf{K}_{1}$.

In summary, we have shown that each of the monomials $f(q)\left(q-q^{-1}\right)^{m_{0}} \mathcal{M}$ in $\mathbf{K}_{m}$ is congruent modulo $\mathbf{K}_{m+1}$ to

$$
f(1)\left(q-q^{-1}\right)^{m_{0}} T_{i_{1} j_{1}}^{\left(0, m_{1}\right)} \cdots T_{i_{k}, j_{k}}^{\left(0, m_{k}\right)}
$$

up to a sign. The image modulo $\mathbf{K}_{m+1}$ of this element is

$$
f(1) \hbar^{m_{0}} \xi_{i_{1} j_{1}}^{\left(0, m_{1}\right)} \cdots \xi_{i_{k} j_{k}}^{\left(0, m_{k}\right)}
$$

and this is in the image of $\varphi$ by definition. This completes the proof that $\varphi$ is surjective.

To prove that $\varphi$ is injective, it is enough to show that the basis of $Y\left(\mathfrak{g l}_{N}\right)$ given by ordered monomials (for some fixed order) in the generators $t_{i j}^{(m)}$ is mapped via $\varphi$ to some linearly independent set in $\tilde{Y}\left(\mathfrak{g l}_{N}\right)$.

By definition, any two ordered monomials $\hbar^{m_{0}} t_{i_{1} j_{1}}^{\left(m_{1}+1\right)} \cdots t_{i_{a} j_{a}}^{\left(m_{a}+1\right)}$ and $\hbar^{n_{0}} t_{k_{1} l_{1}}^{\left(n_{1}+1\right)} \cdots t_{k_{b} l_{b}}^{\left(n_{b}+1\right)}$ with each $m_{d}, n_{d} \geq 0$ and $m_{0}+m_{1}+\cdots+m_{a} \neq n_{0}+n_{1}+\cdots+n_{b}$ are mapped via $\varphi$ to distinct graded pieces in $\widetilde{Y}\left(\mathfrak{g l}_{N}\right)$. It therefore suffices to show that for each fixed $m$, the images under $\varphi$ of all the ordered monomials $\hbar^{m_{0}} t_{i_{1} j_{1}}^{\left(m_{1}+1\right)} \cdots t_{i_{a} j_{a}}^{\left(m_{a}+1\right)}$ with $m_{1}+\cdots+m_{a}=m$ are linearly independent in $\mathbf{K}_{m} / \mathbf{K}_{m+1}$.

Consider any linear combination over $\mathbb{C}$ of ordered monomials $\left(\overline{q-q^{-1}}\right)^{m_{0}} \xi_{i_{1} j_{1}}^{\left(0, m_{1}\right)} \cdots \xi_{i_{a} j_{a}}^{\left(0, m_{a}\right)}$ with $m_{0}+m_{1}+\cdots+m_{a}=m$, and suppose that this sum is zero in $\mathbf{K}_{m} / \mathbf{K}_{m+1}$. Then we have a linear combination $S$ of ordered monomials $\left(q-q^{-1}\right)^{m_{0}} T_{i_{1} j_{1}}^{\left(0, m_{1}\right)} \cdots T_{i_{a} j_{a}}^{\left(0, m_{a}\right)}$ which is not just in $\mathbf{K}_{m}$, but also in $\mathbf{K}_{m+1}$. We can assume that the minimum of the exponents $m_{0}$ is 0 .

$\psi(S)$ is a linear combination of some of the ordered monomials $E_{i_{1} j_{1}}(s-1)^{m_{1}} \cdots E_{i_{a} j_{a}}(s-1)^{m_{a}}$. On the other hand, since $S$ is also in $\mathbf{K}_{m+1}, \psi(S)$ can be expressed as a linear combination of monomials of the form $E_{k_{1} l_{1}} s^{r_{1}}(s-1)^{n_{1}} \cdots E_{k_{b} l_{b}} s^{r_{b}}(s-1)^{n_{b}}$ with $r_{1}, \ldots, r_{b} \in \mathbb{Z}$ and $n_{1}+\cdots+n_{b} \geq$ $m+1$. If $r_{1}=\cdots=r_{b}=0$, then this is impossible unless the coefficients of both linear combinations vanish. Let's prove that the same is true more generally.

For each $r \geq 1$, we have a composite of algebra homomorphisms

$$
\mathfrak{U}\left(\mathcal{L}\left(\mathfrak{g l}_{N}\right)\right) \stackrel{\Delta}{\rightarrow} \mathfrak{U}\left(\mathcal{L}\left(\mathfrak{g l}_{N}\right)\right)^{\otimes r} \stackrel{f^{\otimes r}}{\rightarrow} \operatorname{End}_{\mathbb{C}}\left(\mathbb{C}^{N}\right)^{\otimes r} \otimes \mathbb{C}\left[x_{1}^{ \pm 1}, x_{2}^{ \pm 1}, \ldots, x_{r}^{ \pm 1}\right],
$$

where $\Delta$ is the standard coproduct on the enveloping algebra of a Lie algebra and the homomorphism $f: \mathfrak{U}\left(\mathcal{L}\left(\mathfrak{g l}_{N}\right)\right) \rightarrow \operatorname{End}_{\mathbb{C}}\left(\mathbb{C}^{N}\right) \otimes \mathbb{C}\left[x, x^{-1}\right]$ is given by $f\left(E_{i j} x^{t}\right)=E_{i j} \otimes x^{t}$.

We also have for each choice of $r$ nonnegative integers $\alpha_{1}, \ldots, \alpha_{r}$ a differential operator

$$
\partial_{\alpha_{1}, \ldots, \alpha_{r}}: \operatorname{End}_{\mathbb{C}}\left(\mathbb{C}^{N}\right)^{\otimes r} \otimes \mathbb{C}\left[x_{1}^{ \pm 1}, x_{2}^{ \pm 1}, \ldots, x_{r}^{ \pm 1}\right] \rightarrow \operatorname{End}_{\mathbb{C}}\left(\mathbb{C}^{N}\right)^{\otimes r}
$$


given by

$$
\partial_{\alpha_{1}, \ldots, \alpha_{r}}=\left.\frac{\partial^{\alpha_{1}}}{\partial x_{1}^{\alpha_{1}}} \frac{\partial^{\alpha_{2}}}{\partial x_{2}^{\alpha_{2}}} \cdots \frac{\partial^{\alpha_{r}}}{\partial x_{r}^{\alpha_{r}}}\right|_{x_{1}, \ldots, x_{r}=1} .
$$

Take $r \geq \max \{a, b\}$ where the maximum is taken over all the monomials in $\psi(S)$ (with $a$ and $b$ related to the monomials in $S$ and $\psi(S)$ as above) and note that for any choice of $\alpha_{1}, \ldots, \alpha_{r}$ with $\alpha_{1}+\cdots+\alpha_{r}=m, \psi(S)$ is in the kernel of the composite $\partial_{\alpha_{1}, \ldots, \alpha_{r}} \circ f^{\otimes r} \circ \Delta$ because $n_{1}+\cdots+n_{b} \geq m+1$.

On the other hand, if $S$ is nonzero, then we can find some $\alpha_{1}, \ldots, \alpha_{r}$ such that $\alpha_{1}+\cdots+\alpha_{r}=$ $m$ and $\psi(S)$ is not in the kernel of $\partial_{\alpha_{1}, \ldots, \alpha_{r}} \circ f^{\otimes r} \circ \Delta$ : just choose any of the ordered monomials $E_{i_{1} j_{1}}(s-1)^{m_{1}} \cdots E_{i_{a} j_{a}}(s-1)^{m_{a}}$ in $\psi(S)$ and set $\alpha_{1}=m_{1}, \ldots, \alpha_{a}=m_{a}$ and $\alpha_{d}=0$ for $d>a$.

We have just obtained a contradiction, so $S=0$ and the linear sum of ordered monomials $\xi_{i_{1} j_{1}}^{\left(0, m_{1}\right)} \cdots \xi_{i_{a} j_{a}}^{\left(0, m_{a}\right)}$ must in fact be trivial, as desired.

\section{Twisted quantum loop algebras and Yangians of type $\mathfrak{o}_{N}$ and $\mathfrak{s p}_{N}$}

We will now prove an analogue of Theorem 2.2 for certain twisted Yangians and twisted quantum loop algebras associated to the symmetric pairs $\left(\mathfrak{g l}_{N}, \mathfrak{o}_{N}\right)$ and $\left(\mathfrak{g l}_{N}, \mathfrak{s p}_{N}\right)$. We will treat these two cases simultaneously and denote by $\mathfrak{g}_{N}$ either $\mathfrak{o}_{N}$ or $\mathfrak{s p}_{N}$. Whenever we use the symbols \pm or $\mp$, it is understood that the sign on the top is used for $\mathfrak{g}_{N}=\mathfrak{o}_{N}$ and the sign on the bottom is used for $\mathfrak{g}_{N}=\mathfrak{s p}_{N}$. In this section, we will use $t$ to denote transposition in the first factor of a tensor product of matrices.

In the orthogonal case, let $G=\left(g_{i j}\right)$ be the $N \times N$ identity matrix. For the symplectic case, we take

$$
G=\sum_{k=1}^{N / 2}\left(E_{2 k-1,2 k}-E_{2 k, 2 k-1}\right),
$$

which makes sense since $\mathfrak{s p}_{N}$ is only defined when $N$ is even. Similarly, let $B=\left(b_{i j}\right)$ be the $N \times N$ identity matrix in the orthogonal case, while in the symplectic case we take

$$
B=\sum_{k=1}^{N / 2}\left(q E_{2 k-1,2 k}-E_{2 k, 2 k-1}\right) .
$$

Definition 3.1. Let $R(u)$ be as given in Definition 2.1. The twisted Yangian $Y^{t w}\left(\mathfrak{g}_{N}\right)$ is the unital associative algebra over $\mathbb{C}[\hbar]$ generated by $\left\{s_{i j}^{(r)} \mid r \in \mathbb{Z}_{+}, i, j=1, \ldots, N\right\}$ where $s_{i j}^{(0)}=g_{i j}$, together with the following relations: if $s_{i j}(u)=\sum_{r=0}^{\infty} s_{i j}^{(r)} u^{-r}$ and $s(u)=\sum_{i, j=1}^{N} E_{i j} \otimes s_{i j}(u)$, then

$$
R(u-v) s_{1}(u) R^{t}(-u-v) s_{2}(v)=s_{2}(v) R^{t}(-u-v) s_{1}(u) R(u-v)
$$

and

$$
s^{t}(-u)= \pm s(u)+\hbar \frac{s(u)-s(-u)}{2 u},
$$

where $s_{1}(u)\left(\right.$ resp. $\left.s_{2}(u)\right)$ is the element of $\operatorname{End}_{\mathbb{C}}\left(\mathbb{C}^{N}\right) \otimes \operatorname{End}_{\mathbb{C}}\left(\mathbb{C}^{N}\right) \otimes Y^{t w}\left(\mathfrak{g}_{N}\right)\left[\left[u^{-1}\right]\right]$ obtained by replacing $E_{i j}$ by $E_{i j} \otimes I$ (resp. $\left.I \otimes E_{i j}\right)$ in $s(u)$. 
The twisted Yangian is a deformation of the universal enveloping algebra of the twisted current algebra $\mathfrak{g}_{N}^{t w}[s]$ which is defined in the following way.

Definition 3.2. Let $\sigma$ be the automorphism of $\mathfrak{g l}_{N}$ given by

$$
\sigma\left(E_{i j}\right)=-E_{j i}
$$

if $\mathfrak{g}_{N}=\mathfrak{o}_{N}$, while

$$
\sigma\left(E_{i j}\right)=(-1)^{i+j-1} E_{j^{\prime} i^{\prime}}
$$

if $\mathfrak{g}_{N}=\mathfrak{s p}_{N}$. Here, $i^{\prime}=i-1$ if $i$ is even and $i^{\prime}=i+1$ is $i$ is odd. The twisted current algebra is the subalgebra of $\mathfrak{g l}_{N}[s]$ given by

$$
\mathfrak{g}_{N}^{t w}[s]=\left\{A(s) \in \mathfrak{g l}_{N}[s] \mid \sigma(A(s))=A(-s)\right\} .
$$

The twisted Yangians can be regarded as subalgebras of the Yangian for $\mathfrak{g l}_{N}$ :

Proposition 3.1 ([31]). The assignment

$$
s_{i j}^{(r)} \mapsto \sum_{k=1}^{N}\left(g_{k j} t_{i k}^{(r)}+(-1)^{r} g_{i k} t_{j k}^{(r)}\right)+\hbar \sum_{k, l=1}^{N} \sum_{p=1}^{r-1}(-1)^{r-p} g_{k l} t_{i k}^{(p)} t_{j l}^{(r-p)}
$$

provides an embedding of $Y^{t w}\left(\mathfrak{g}_{N}\right)$ into $Y\left(\mathfrak{g l}_{N}\right)$.

Definition 3.3 ([29]). The twisted quantum loop algebra $\mathfrak{U}_{q}\left(\mathcal{L}^{t w}\left(\mathfrak{o}_{N}\right)\right)$ is the unital associative algebra over $\mathbb{C}(q)$ generated by $\left\{S_{i j}^{(r)} \mid r \in \mathbb{Z}_{+}, 1 \leq i, j \leq N\right\}$ which are subject to the relations

$$
\begin{aligned}
& S_{i j}^{(0)}=0 \quad \text { if } \quad 1 \leq i<j \leq n, \quad S_{i i}^{(0)}=1 \quad \forall 1 \leq i \leq n, \\
& R_{q}(u, v) S_{1}(u) R_{q}^{t}\left(u^{-1}, v\right) S_{2}(v)=S_{2}(v) R_{q}^{t}\left(u^{-1}, v\right) S_{1}(u) R_{q}(u, v),
\end{aligned}
$$

where $S_{a}(u)$ is obtained from $S(u)$ in the same way as in Definition 3.1, except that in this case we have $S(u):=\sum_{i, j=1}^{N} E_{i j} \otimes S_{i j}(u)$ and $S_{i j}(u):=\sum_{r=0}^{\infty} S_{i j}^{(r)} u^{-r}$.

Definition 3.4 ([29]). The twisted quantum loop algebra $\mathfrak{U}_{q}\left(\mathcal{L}^{t w}\left(\mathfrak{s p}_{N}\right)\right)$ is the unital associative algebra over $\mathbb{C}(q)$ with generators $\left\{S_{i j}^{(r)} \mid r \in \mathbb{Z}_{+}, 1 \leq i, j \leq N\right\}$ and $\left\{\left(S_{i i^{\prime}}^{(0)}\right)^{-1} \mid i=\right.$ $1,3, \ldots, N-1\}$, which are subject to the relations

$$
\begin{aligned}
& S_{i j}^{(0)}=0 \quad \text { whenever } i<j \quad \text { and } \quad j \neq i^{\prime} \\
& S_{i^{\prime} i^{\prime}}^{(0)} S_{i i}^{(0)}-q^{2} S_{i^{\prime} i}^{(0)} S_{i i^{\prime}}^{(0)}=q^{3}, \quad i=1,3, \ldots, N-1, \\
& S_{i i^{\prime}}^{(0)}\left(S_{i i^{\prime}}^{(0)}\right)^{-1}=\left(S_{i i^{\prime}}^{(0)}\right)^{-1} S_{i i^{\prime}}^{(0)}=1, \quad i=1,3, \ldots, N-1, \\
& R_{q}(u, v) S_{1}(u) R_{q}^{t}\left(u^{-1}, v\right) S_{2}(v)=S_{2}(v) R_{q}^{t}\left(u^{-1}, v\right) S_{1}(u) R_{q}(u, v),
\end{aligned}
$$

where $S_{a}(u)$ is defined here in the same way as in Definition 3.3.

The twisted quantum loop algebra $\mathfrak{U}_{q}\left(\mathcal{L}^{t w}\left(\mathfrak{g}_{N}\right)\right)$ is a deformation of the universal enveloping algebra of the twisted loop algebra $\mathfrak{g}_{N}^{t w}\left[s, s^{-1}\right]$ which is defined in the following way:

Definition 3.5. The twisted loop algebra $\mathfrak{g}_{N}^{t w}\left[s, s^{-1}\right]$ is the Lie subalgebra of $\mathcal{L}\left(\mathfrak{g l}_{N}\right)$ given by

$$
\mathfrak{g}_{N}^{t w}\left[s, s^{-1}\right]=\left\{A(s) \in \mathcal{L}\left(\mathfrak{g l}_{N}\right) \mid \sigma(A(s))=A\left(s^{-1}\right)\right\},
$$

where $\sigma$ is given by (3.1) for $\mathfrak{g}_{N}=\mathfrak{o}_{N}$ and by (3.2) for $\mathfrak{g}_{N}=\mathfrak{s p}_{N}$. This algebra is also denoted by $\mathcal{L}^{t w}\left(\mathfrak{g}_{N}\right)$. 
The twisted quantum loop algebras can be regarded as subalgebras of $\mathfrak{U}_{q}\left(\mathcal{L}\left(\mathfrak{g l}_{N}\right)\right)$ :

Proposition 3.2 ([29]). The assignment

$$
S_{i j}^{(r)} \mapsto \sum_{k, l=1}^{N} \sum_{p=0}^{r} b_{k l} T_{i k}^{(p)} \bar{T}_{j l}^{(r-p)}
$$

provides an embedding of $\mathfrak{U}_{q}\left(\mathcal{L}^{t w}\left(\mathfrak{g}_{N}\right)\right)$ into $\mathfrak{U}_{q}\left(\mathcal{L}\left(\mathfrak{g l}_{N}\right)\right)$.

For each $r>0$, let $S_{i j}^{(r, 0)}=\frac{S_{i j}^{(r)}}{q-q^{-1}}$. When $\mathfrak{g}_{N}=\mathfrak{o}_{N}$, we set $S_{i j}^{(0,0)}=\frac{S_{i j}^{(0)}}{q-q^{-1}}$ when $i>j$ and $S_{i j}^{(0,0)}=-S_{j i}^{(0,0)}$ when $i \leq j$; when $\mathfrak{g}_{N}=\mathfrak{s p}_{N}$, we set $S_{i j}^{(0,0)}=\frac{S_{i j}^{(0)}-b_{i j}}{q-q^{-1}}$ when $i \geq j$ or $j=i^{\prime}$ and $S_{i j}^{(0,0)}=-S_{j i}^{(0,0)}$ when $i<j$ and $j \neq i^{\prime}$.

We define inductively $S_{i j}^{(r, m)}$ by

$$
S_{i j}^{(r, m+1)}=S_{i j}^{(r+1, m)}-S_{i j}^{(r, m)}
$$

for $m \geq 0$.

Lemma 3.1. Identify $S_{i j}^{(r)}$ with its image under the embeddings of Proposition 3.2. For any $r>0$ and $m \geq 0$, we have

$$
\begin{aligned}
S_{i j}^{(r, m)}= & \sum_{k=1}^{N}\left(b_{k j} T_{i k}^{(r, m)}+b_{i k} \bar{T}_{j k}^{(r, m)}\right)+\left(q-q^{-1}\right) \sum_{k, l=1}^{N} \sum_{p=1}^{r-1} b_{k l} T_{i k}^{(p, 0)} \bar{T}_{j l}^{(r-p, m)} \\
& +\left(q-q^{-1}\right)\left(\sum_{\substack{1 \leq k \leq i \\
1 \leq l \leq N}}\left(\frac{q}{q+1}\right)^{\delta_{i k}} b_{k l} T_{i k}^{(0,0)} \bar{T}_{j l}^{(r, m)}+\sum_{\substack{1 \leq k \leq N \\
j \leq l \leq N}}\left(\frac{q}{q+1}\right)^{\delta_{j l}} b_{k l} T_{i k}^{(r, m)} \bar{T}_{j l}^{(0,0)}\right) \\
& +\left(q-q^{-1}\right) \sum_{k, l=1}^{N} \sum_{a+b=m-1} b_{k l} T_{i k}^{(r, a)} \bar{T}_{j l}^{(1, b)} .
\end{aligned}
$$

Proof. Since $T_{i k}^{(0)}=0$ when $i<k$ and $\bar{T}_{j l}^{(0)}=0$ when $j>l$, we have by definition

$$
\begin{aligned}
S_{i j}^{(r, 0)}= & \left(q-q^{-1}\right) \sum_{k, l=1}^{N} \sum_{p=1}^{r-1} b_{k l}\left(\frac{T_{i k}^{(p)}}{q-q^{-1}}\right)\left(\frac{\bar{T}_{j l}^{(r-p)}}{q-q^{-1}}\right) \\
& +\left(q-q^{-1}\right) \sum_{\substack{1 \leq k<i \\
1 \leq l \leq N}} b_{k l}\left(\frac{T_{i k}^{(0)}}{q-q^{-1}}\right)\left(\frac{\bar{T}_{j l}^{(r)}}{q-q^{-1}}\right) \\
& +(q-1) \sum_{l=1}^{N} b_{i l}\left(\frac{T_{i i}^{(0)}-1}{q-1}\right)\left(\frac{\bar{T}_{j l}^{(r)}}{q-q^{-1}}\right)+\sum_{l=1}^{N} b_{i l} \frac{\bar{T}_{j l}^{(r)}}{q-q^{-1}} \\
& +\left(q-q^{-1}\right) \sum_{\substack{1 \leq k \leq N \\
j<l \leq N}} b_{k l}\left(\frac{T_{i k}^{(r)}}{q-q^{-1}}\right)\left(\frac{\bar{T}_{j l}^{(0)}}{q-q^{-1}}\right) \\
& +(q-1) \sum_{k=1}^{N} b_{k j}\left(\frac{T_{i k}^{(r)}}{q-q^{-1}}\right)\left(\frac{\bar{T}_{j j}^{(0)}-1}{q-1}\right)+\sum_{k=1}^{N} b_{k j} \frac{T_{i k}^{(r)}}{q-q^{-1}}
\end{aligned}
$$




$$
\begin{aligned}
= & \sum_{k=1}^{N}\left(b_{k j} T_{i k}^{(r, 0)}+b_{i k} \bar{T}_{j k}^{(r, 0)}\right)+\left(q-q^{-1}\right) \sum_{k, l=1}^{N} \sum_{p=1}^{r-1} b_{k l} T_{i k}^{(p, 0)} \bar{T}_{j l}^{(r-p, 0)} \\
& +\left(q-q^{-1}\right)\left(\sum_{\substack{1 \leq k \leq i \\
1 \leq l \leq N}}\left(\frac{q}{q+1}\right)^{\delta_{i k}} b_{k l} T_{i k}^{(0,0)} \bar{T}_{j l}^{(r, 0)}+\sum_{\substack{1 \leq i \leq N \\
j \leq l \leq N}}\left(\frac{q}{q+1}\right)^{\delta_{j l}} b_{k l} T_{i k}^{(r, 0)} \bar{T}_{j l}^{(0,0)}\right) .
\end{aligned}
$$

This proves the case $m=0$. The case $m=1$ is similar, except for the presence of an extra sum. The general case follows immediately by induction on $m$.

The previous lemma along with (2.7) yields the next corollary.

Corollary 3.1. Under the same assumption as in the previous lemma, we have $S_{i j}^{(r, m)} \in \mathbf{K}_{m}$, and

$$
\begin{aligned}
S_{i j}^{(r, m)} \equiv & \sum_{k=1}^{N}\left(b_{k j} T_{i k}^{(0, m)}+(-1)^{m+1} b_{i k} T_{j k}^{(0, m)}\right) \\
& +\left(q-q^{-1}\right) \sum_{k, l=1}^{N} \sum_{p=1}^{m}(-1)^{m+1-p} b_{k l} T_{i k}^{(0, p-1)} T_{j l}^{(0, m-p)}
\end{aligned}
$$

modulo $\mathbf{K}_{m+1}$.

Again let's view $S_{i j}^{(r)}$ as an element of $\mathfrak{U}_{q}\left(\mathcal{L}\left(\mathfrak{g l}_{N}\right)\right)$. Let $\zeta_{i j}^{(r, m)}$ be the image of $S_{i j}^{(r, m)}$ in $\mathbf{K}_{m} / \mathbf{K}_{m+1}$. Note that, by Corollary $3.1, \zeta_{i j}^{(r, m)}$ is independent of $r>0$.

Theorem 3.1. Identify $s_{i j}^{(m+1)}$ with its image under the embedding of Proposition 3.1. For each $m \geq 0$, we have

$$
\varphi\left(s_{i j}^{(m+1)}\right)=\zeta_{i j}^{(1, m)}
$$

where $\varphi$ is the isomorphism of Theorem 2.2.

Remark 3.1. It would also be possible to prove a similar theorem with $\zeta_{i j}^{(0, m)}$ instead of $\zeta_{i j}^{(1, m)}$, but the proof would be longer because we would have to consider different cases in order to take into account that about half of the generators $S_{i j}^{(0)}$ are 0 .

Proof. By Proposition 3.1, Corollary 3.1 and the definition of $\varphi$, we have

$$
\begin{aligned}
\varphi\left(s_{i j}^{(m+1)}\right)= & \sum_{k=1}^{N}\left(g_{k j} \xi_{i k}^{(0, m)}+(-1)^{m+1} g_{i k} \xi_{j k}^{(0, m)}\right) \\
& +\hbar \sum_{k, l=1}^{N} \sum_{p=1}^{m}(-1)^{m+1-p} g_{k l} \xi_{i k}^{(0, p-1)} \xi_{j l}^{(0, m-p)}=\zeta_{i j}^{(1, m)},
\end{aligned}
$$

where we have used the fact that $b_{k l}-g_{k l}$ is divisible by $q-1$.

We can obtain an analogue of Theorem 2.2. From Theorem 2.1 and Proposition 3.2, we can deduce that the enveloping algebra of $\mathfrak{g}_{N}^{t w}[s]$ is the limit when $q \rightarrow 1$ of $\mathfrak{U}_{q}\left(\mathcal{L}^{t w}\left(\mathfrak{g}_{N}\right)\right)$ in the following sense: if we let $\mathfrak{U}_{\mathcal{A}}\left(\mathcal{L}^{t w}\left(\mathfrak{g}_{N}\right)\right)$ be the $\mathcal{A}$-subalgebra of $\mathfrak{U}_{q}\left(\mathcal{L}^{t w}\left(\mathfrak{g}_{N}\right)\right)$ generated by the $S_{i j}^{(r, 0)}$ for all $i, j$ and all $r \geq 0$, then $\mathfrak{U}_{\mathcal{A}}\left(\mathcal{L}^{t w}\left(\mathfrak{g}_{N}\right)\right) /(q-1) \mathfrak{U}_{\mathcal{A}}\left(\mathcal{L}^{t w}\left(\mathfrak{g}_{N}\right)\right)$ is isomorphic to $\mathfrak{U}\left(\mathfrak{g}_{N}^{t w}\left[s, s^{-1}\right]\right)$ (see the proof of Corollaries 3.5 and 3.12 in [29]). 
We can define an algebra $\tilde{Y}^{t w}\left(\mathfrak{g}_{N}\right)$ similarly to how we defined $\tilde{Y}\left(\mathfrak{g l}_{N}\right)$. For $m \geq 0$, in the orthogonal case, denote by $\mathrm{K}_{m}^{t w}$ the Lie ideal of $\mathfrak{o}_{N}^{t w}\left[s, s^{-1}\right]$ spanned by

$$
E_{i j} s^{r}(s-1)^{m}-E_{j i} s^{-r}\left(s^{-1}-1\right)^{m}
$$

for all $r \in \mathbb{Z}$. In the symplectic case, let $\mathrm{K}_{m}^{t w}$ be the Lie ideal of $\mathfrak{s p}_{N}^{t w}\left[s, s^{-1}\right]$ spanned by

$$
E_{i j^{\prime}} s^{r}(s-1)^{m}-(-1)^{i+j+1} E_{j i^{\prime}} s^{-r}\left(s^{-1}-1\right)^{m}
$$

for all $r \in \mathbb{Z}$. Let $U^{t w}$ be the subspace of $\mathfrak{U}_{\mathcal{A}}\left(\mathcal{L}^{t w}\left(\mathfrak{g}_{N}\right)\right)$ spanned over $\mathbb{C}$ by all the generators $S_{i j}^{(r, 0)}$, and observe that $U^{t w} \cap(q-1) \mathfrak{U}_{\mathcal{A}}\left(\mathcal{L}^{t w}\left(\mathfrak{g}_{N}\right)\right)=\{0\}$. Let $\mathbb{K}_{m}^{t w}$ be the two-sided ideal of $\mathfrak{U}_{\mathcal{A}}\left(\mathcal{L}^{t w}\left(\mathfrak{g}_{N}\right)\right)$ generated by $\psi^{-1}\left(\mathrm{~K}_{m}^{t w}\right) \cap U^{t w}$, where $\psi$ this time denotes the composite

$$
\mathfrak{U}_{\mathcal{A}}\left(\mathcal{L}^{t w}\left(\mathfrak{g}_{N}\right)\right) \rightarrow \mathfrak{U}_{\mathcal{A}}\left(\mathcal{L}^{t w}\left(\mathfrak{g}_{N}\right)\right) /(q-1) \mathfrak{U}_{\mathcal{A}}\left(\mathcal{L}^{t w}\left(\mathfrak{g}_{N}\right)\right) \stackrel{\sim}{\longrightarrow} \mathfrak{U}\left(\mathfrak{g}_{N}^{t w}\left[s, s^{-1}\right]\right) .
$$

Set $\mathbf{K}_{m}^{t w}$ equal to the sum of the ideals $\left(q-q^{-1}\right)^{m_{0}} \mathbb{K}_{m_{1}}^{t w} \cdots \mathbb{K}_{m_{k}}^{t w}$ with $m_{0}+m_{1}+\cdots+m_{k} \geq m$.

Let $\widetilde{Y}^{t w}\left(\mathfrak{g}_{N}\right)$ be the $\mathbb{C}$-algebra

$$
\bigoplus_{m=0}^{\infty} \mathbf{K}_{m}^{t w} / \mathbf{K}_{m+1}^{t w}
$$

where $\mathbf{K}_{0}^{t w}=\mathfrak{U}_{\mathcal{A}}\left(\mathcal{L}^{t w}\left(\mathfrak{g}_{N}\right)\right)$. We also view $\widetilde{Y}^{t w}\left(\mathfrak{g}_{N}\right)$ as an algebra over $\mathbb{C}[\hbar]$ by setting $\hbar=$ $\overline{q-q^{-1}} \in \mathbf{K}_{1}^{t w} / \mathbf{K}_{2}^{t w}$.

Theorem 3.2. $Y^{t w}\left(\mathfrak{g}_{N}\right)$ is isomorphic to $\widetilde{Y}^{t w}\left(\mathfrak{g}_{N}\right)$ via the function $\varphi^{t w}$ that sends $s_{i j}^{(m+1)}$ to $\overline{S_{i j}^{(1, m)}} \in \mathbf{K}_{m}^{t w} / \mathbf{K}_{m+1}^{t w}$ for $m \geq 0$.

Proof. Theorem 3.1 implies that the following diagram is commutative:

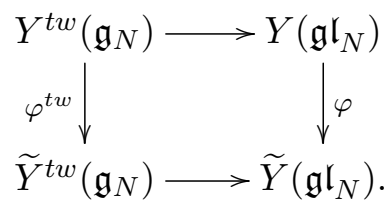

In this diagram, the top horizontal arrow is the embedding of Proposition 3.1 and the bottom horizontal arrow is the one induced from the embedding of Proposition 3.2. The injectivity of $\varphi^{t w}$ now follows from the fact that $\varphi$ provides an isomorphism between $Y\left(\mathfrak{g l}_{N}\right)$ and $\widetilde{Y}\left(\mathfrak{g l}_{N}\right)$ : see Theorem 2.2.

We need to see that $\varphi^{t w}$ is surjective. Define elements $\widetilde{S}_{i j}^{(r, m)}$ with $0 \leq r \leq m$ as follows: for each $m \geq 0$, let $\widetilde{S}_{i j}^{(0, m)}=S_{j i}^{(0, m)}$ and $\widetilde{S}_{i j}^{(m, m)}=(-1)^{m+1} S_{i j}^{(0, m)}$, and for $1 \leq r \leq m$ let

$$
\widetilde{S}_{i j}^{(r, m+1)}=\widetilde{S}_{i j}^{(r-1, m)}-\widetilde{S}_{i j}^{(r, m)} .
$$

Then induction on $m$ shows that

$$
\begin{aligned}
& \psi\left(S_{i j}^{(r, m)}\right)=E_{i j} s^{r}(s-1)^{m}-E_{j i} s^{-r}\left(s^{-1}-1\right)^{m}, \\
& \psi\left(S_{j i}^{(r, m)}\right)=(-1)^{m+1}\left(E_{i j} s^{-(m+r)}(s-1)^{m}-E_{j i} s^{m+r}\left(s^{-1}-1\right)^{m}\right), \\
& \psi\left(\widetilde{S}_{i j}^{(r, m)}\right)=(-1)^{m+1}\left(E_{i j} s^{-(m-r)}(s-1)^{m}-E_{j i} s^{m-r}\left(s^{-1}-1\right)^{m}\right) .
\end{aligned}
$$


in the orthogonal case, and similarly in the symplectic case. It follows that for any fixed $m$, the images of these elements under $\psi$ span $\mathrm{K}_{m}^{t w}$, and they are all in $U^{t w}$ by definition. Now note that for any element $X \in \psi^{-1}\left(\mathrm{~K}_{m}^{t w}\right) \cap U^{t w}$, there is some element $Y$ in

$$
\operatorname{span}_{\mathbb{C}}\left\{S_{i j}^{(r, m)}, \widetilde{S}_{i j}^{(r, m)} \mid i, j=1, \ldots, N, r \in \mathbb{Z}_{\geq 0}\right\}
$$

for which $X-Y \in(q-1) \mathfrak{U}_{\mathcal{A}}\left(\mathcal{L}^{t w}\left(\mathfrak{g}_{N}\right)\right)$. This follows from the fact that

$$
\mathfrak{U}_{\mathcal{A}}\left(\mathcal{L}^{t w}\left(\mathfrak{g}_{N}\right)\right) /(q-1) \mathfrak{U}_{\mathcal{A}}\left(\mathcal{L}^{t w}\left(\mathfrak{g}_{N}\right)\right) \stackrel{\sim}{\longrightarrow} \mathfrak{U}\left(\mathfrak{g}_{N}^{t w}\left[s, s^{-1}\right]\right)
$$

is an isomorphism. Since $X-Y$ is also in $U^{t w}$ and since $U^{t w} \cap(q-1) \mathfrak{U}_{\mathcal{A}}\left(\mathcal{L}^{t w}\left(\mathfrak{g}_{N}\right)\right)=\{0\}$, we see that $X=Y$. Therefore, any element of $\mathbf{K}_{m}^{t w}$ is a sum of monomials $f(q)\left(q-q^{-1}\right)^{m_{0}} \mathcal{M}$, where $f(q) \in \mathcal{A}$ is not divisible by $q-1$ and $\mathcal{M}=\sigma_{i_{1} j_{1}}^{\left(r_{1}, m_{1}\right)} \cdots \sigma_{i_{k} j_{k}}^{\left(r_{k}, m_{k}\right)}$ with

$$
\sigma_{i_{d} j_{d}}^{\left(r_{d}, m_{d}\right)} \in\left\{S_{i_{d} j_{d}}^{\left(r_{d}, m_{d}\right)}, \widetilde{S}_{i_{d} j_{d}}^{\left(r_{d}, m_{d}\right)}\right\}
$$

and $m_{0}+\cdots+m_{k} \geq m$. Following the same argument as in the $\mathfrak{g l}_{N}$ case, such a monomial is congruent modulo $\mathbf{K}_{m+1}^{t w}$ to

$$
f(1)\left(q-q^{-1}\right)^{m_{0}} S_{i_{1} j_{1}}^{\left(1, m_{1}\right)} \cdots S_{i_{k} j_{k}}^{\left(1, m_{k}\right)}
$$

up to a sign. The image modulo $\mathbf{K}_{m+1}^{t w}$ of this element is

$$
f(1) \hbar^{m_{0}} \overline{S_{i_{1} j_{1}}^{\left(1, m_{1}\right)}} \cdots \overline{S_{i_{k} j_{k}}^{\left(1, m_{k}\right)}}
$$

and this is in the image of $\varphi^{t w}$, which proves that $\varphi^{t w}$ is surjective.

\section{Acknowledgements}

The research of N. Guay was supported by a Discovery Grant from the Natural Sciences and Engineering Research Council of Canada. P. Conner was supported by a Postgraduate Scholarship CGS-M from the same agency.

\section{References}

[1] Arnaudon D., Molev A., Ragoucy E., On the $R$-matrix realization of Yangians and their representations, Ann. Henri Poincaré 7 (2006), 1269-1325, math.QA/0511481.

[2] Bernard D., Hidden Yangians in 2D massive current algebras, Comm. Math. Phys. 137 (1991), $191-208$.

[3] Bernard D., LeClair A., Quantum group symmetries and nonlocal currents in 2D QFT, Comm. Math. Phys. 142 (1991), 99-138.

[4] Bernard D., Maassarani Z., Mathieu P., Logarithmic Yangians in WZW models, Modern Phys. Lett. A 12 (1997), 535-544, hep-th/9612217.

[5] Brundan J., Kleshchev A., Parabolic presentations of the Yangian $Y\left(\mathfrak{g l}_{n}\right)$, Comm. Math. Phys. 254 (2005), 191-220, math.QA/0407011.

[6] Chari V., Pressley A., Yangians, integrable quantum systems and Dorey's rule, Comm. Math. Phys. 181 (1996), 265-302, hep-th/9505085.

[7] Chen H., Guay N., Twisted affine Lie superalgebra of type $Q$ and quantization of its enveloping superalgebra, Math. Z. 272 (2012), 317-347.

[8] Chen H., Guay N., Ma X., Twisted Yangians, twisted quantum loop algebras and affine Hecke algebras of type BC, Trans. Amer. Math. Soc. 366 (2014), 2517-2574.

[9] Cherednik I.V., A new interpretation of Gelfand-Tzetlin bases, Duke Math. J. 54 (1987), 563-577. 
[10] De La Rosa Gomez A., MacKay N.J., Twisted Yangian symmetry of the open Hubbard model, J. Phys. A: Math. Theor. 47 (2014), 305203, 9 pages, arXiv:1404.2095.

[11] Delius G.W., MacKay N.J., Short B.J., Boundary remnant of Yangian symmetry and the structure of rational reflection matrices, Phys. Lett. B 522 (2001), 335-344, hep-th/0109115.

[12] Ding J.T., Frenkel I.B., Isomorphism of two realizations of quantum affine algebra $U_{q}(\widehat{\mathfrak{g l}(n)})$, Comm. Math . Phys. 156 (1993), 277-300.

[13] Drinfeld V.G., Quantum groups, in Proceedings of the International Congress of Mathematicians, Vols. 1, 2 (Berkeley, Calif., 1986), Amer. Math. Soc., Providence, RI, 1987, 798-820.

[14] Drinfeld V.G., A new realization of Yangians and of quantum affine algebras, Soviet Math. Dokl. 36 (1988), 212-216.

[15] Gautam S., Toledano Laredo V., Yangians, quantum loop algebras and abelian difference equations, arXiv:1310.7318.

[16] Gautam S., Toledano Laredo V., Meromorphic Kazhdan-Lusztig equivalence for Yangians and quantum loop algebras, arXiv:1403.5251.

[17] Gautam S., Toledano Laredo V., Yangians and quantum loop algebras, Selecta Math. (N.S.) 19 (2013), 271-336, arXiv:1012.3687.

[18] Guay N., Ma X., From quantum loop algebras to Yangians, J. Lond. Math. Soc. 86 (2012), 683-700.

[19] Guay N., Regelskis V., Twisted Yangians for symmetric pairs of types B, C, D, arXiv:1407.5247.

[20] Haldane F.D.M., Ha Z.N.C., Talstra J.C., Bernard D., Pasquier V., Yangian symmetry of integrable quantum chains with long-range interactions and a new description of states in conformal field theory, Phys. Rev. Lett. 69 (1992), 2021-2025.

[21] Kolb S., Quantum symmetric Kac-Moody pairs, Adv. Math. 267 (2014), 395-469, arXiv:1207.6036.

[22] MacKay N., Regelskis V., Achiral boundaries and the twisted Yangian of the D5-brane, J. High Energy Phys. 2011 (2011), no. 8, 019, 22 pages, arXiv:1105.4128.

[23] MacKay N., Regelskis V., Reflection algebra, Yangian symmetry and bound-states in AdS/CFT, J. High Energy Phys. 2012 (2012), no. 1, 134, 31 pages, arXiv:1101.6062.

[24] MacKay N.J., Rational K-matrices and representations of twisted Yangians, J. Phys. A: Math. Gen. 35 (2002), 7865-7876, math.QA/0205155.

[25] MacKay N.J., Introduction to Yangian symmetry in integrable field theory, Internat. J. Modern Phys. A 20 (2005), 7189-7217, hep-th/0409183.

[26] Mintchev M., Ragoucy E., Sorba P., Zaugg Ph., Yangian symmetry in the nonlinear Schrödinger hierarchy, J. Phys. A: Math. Gen. 32 (1999), 5885-5900, hep-th/9905105.

[27] Molev A., Yangians and classical Lie algebras, Mathematical Surveys and Monographs, Vol. 143, Amer. Math. Soc., Providence, RI, 2007.

[28] Molev A., Ragoucy E., Representations of reflection algebras, Rev. Math. Phys. 14 (2002), 317-342, math.QA/0107213.

[29] Molev A., Ragoucy E., Sorba P., Coideal subalgebras in quantum affine algebras, Rev. Math. Phys. 15 (2003), 789-822, math.QA/0208140.

[30] Nazarov M., Yangian of the queer Lie superalgebra, Comm. Math. Phys. 208 (1999), 195-223, math.QA/9902146.

[31] Olshanskii G.I., Twisted Yangians and infinite-dimensional classical Lie algebras, in Quantum Groups (Leningrad, 1990), Lecture Notes in Math., Vol. 1510, Springer, Berlin, 1992, 104-119.

[32] Reshetikhin N.Yu., Takhtadzhyan L.A., Faddeev L.D., Quantization of Lie groups and Lie algebras, Leningrad Math. J. 1 (1990), 193-225. 